\title{
Jab1 is a specificity factor for E2F1-induced apoptosis
}

\author{
Timothy C. Hallstrom and Joseph R. Nevins ${ }^{1}$ \\ Duke Institute for Genome Sciences and Policy Department of Molecular Genetics and Microbiology, Duke University \\ Medical Center, Durham, North Carolina 27710, USA
}

The members of the E2F family of transcription factors are key regulators of genes involved in cell cycle progression, cell fate determination, DNA damage repair, and apoptosis. Many cell-based experiments suggest that E2F1 is a stronger inducer of apoptosis than the other E2Fs. Our previous work identified the E2F1 marked box and flanking region as critical for the specificity in E2F1 apoptosis induction. We have now used a yeast two-hybrid screen to identify proteins that bind the E2F1 marked box and flanking regions, with a potential role in E2F1 apoptosis induction. We identified Jab1 as an E2F1-specific binding protein and showed that Jab1 and E2F1 coexpression synergistically induce apoptosis, coincident with an induction of p53 protein accumulation. In contrast, Jab1 does not synergize with E2F1 to promote cell cycle entry. Cells depleted of Jab1 are deficient for both E2F1-induced apoptosis and induction of p53 accumulation. We suggest that Jab1 is an essential cofactor for the apoptotic function of E2F1.

[Keywords: E2F; Jab1; apoptosis]

Received June 14, 2005; revised version accepted December 28, 2005.

The E2F family of transcription factors is comprised of eight members grouped into several categories based on their sequence domain homology and biological function (Dyson 1998; Nevins 1998; DeGregori 2002; Attwooll et al. 2004). E2F1, E2F2, and E2F3a comprise the E2F transcriptional activator family and share significant sequence/domain homology and functional overlap. Each of these factors can drive quiescent cells into $S$ phase by activating target genes required for the G1/S transition, and conditional elimination of all three activator E2Fs leads to a complete proliferative block (Wu et al. 2001). Despite this functional overlap, both mutant mouse models and cell-based experiments suggest that the activator E2Fs perform many distinct cellular functions. For example, MEFs derived from $E 2 F 3^{-/-}$mutant mice show reduced mitogen-induced activation of E2F targets, consistent with a role for E2F3 in the activation of genes encoding DNA replication proteins (Leone et al. 1998; Humbert et al. 2000). Conversely, E2F1 appears to be a stronger inducer of apoptosis than either E2F2 or E2F3 when expressed in the absence of growth factors (Qin et al. 1994; Shan and Lee 1994; Wu and Levine 1994; Kowalik et al. 1995, 1998; DeGregori et al. 1997). Furthermore, apoptosis triggered by E2F3 expression in vivo requires E2F1 (Denchi and Helin 2005). E2F1 induces apoptosis in part through the transcription of proapoptotic genes such as ARF, p73, APAF1, Casp3, Casp7, and Chk2, and by

${ }^{1}$ Corresponding author.

E-MAIL j.nevins@duke.edu; FAX (919) 681-8973.

Article published online ahead of print. Article and publication date are at http://www.genesdev.org/cgi/doi/10.1101/ gad.1345006. down-regulating expression of the anti-apoptotic genes Traf2 and Mcl1 (Bates et al. 1998; Irwin et al. 2000; Lissy et al. 2000; Stiewe and Putzer 2000; Moroni et al. 2001; Croxton et al. 2002; Nahle et al. 2002; Rogoff et al. 2002, 2004). Furthermore, $E 2 \mathrm{~F}^{-/-}$mice display impaired Tcell apoptosis and are predisposed to cancer, developing a wide range of tumors in adult mice (Field et al. 1996). $\mathrm{Rb}^{-/-}$mice exhibit extensive apoptosis in the developing CNS, lens, and retina (Jacks et al. 1992; Lee et al. 1992), and at least part of this apoptosis is due to E2F1 deregulation (Pan et al. 1998; Tsai et al. 1998; Yamasaki et al. 1998; Ziebold et al. 2001; Saavedra et al. 2002). Additional studies have demonstrated that E2F1, but not E2F2 or E2F3, is phosphorylated by the ATM/ATR and Chk2 kinases in response to DNA damage, leading to stabilization of E2F1 protein levels (Lin et al. 2001; Stevens et al. 2003). These combined findings support the model that E2F1 possesses a unique role among the activator E2Fs in its ability to respond to DNA damage and induce apoptosis.

These observations raise the question: What mechanism distinguishes E2F1 from the other E2F proteins in triggering the apoptotic pathway? To begin to address this question, we generated a series of chimeric proteins that interchange E2F1 and E2F3 domains (Hallstrom and Nevins 2003). These experiments demonstrated that the marked box domain of E2F1 is critical for the ability of E2F1 to specifically induce apoptosis. We observed that whereas E2F3 caused little apoptosis, an E2F3 chimera containing the E2F1 marked box and flanking region strongly induced apoptosis and both $\mathrm{p} 53$ and $\mathrm{p} 73$ protein 
accumulation. Conversely, E2F1 carrying the E2F3 marked box and adjacent region no longer induced apoptosis or p53/p73 accumulation like full-length E2F1. From this, we concluded that the marked box and adjacent region of E2F1 are significantly responsible for its unique ability to strongly induce apoptosis.

Other recent work has implicated a similar function for the marked box region of E2F activator proteins in mediating transcriptional specificity through the association with other transcription factors. These interactions provide a basis for specificity of E2F transcriptional control because the interactions are restricted to particular E2F family members. For example, E2F2 and E2F3 both interact with RYBP (Ring1- and YY1-binding protein), which also binds to the transcription factor YY1 resulting in a trimeric complex (Schlisio et al. 2002). Also, E2F3 interacts with the E box transcription factor TFE3 whereas E2F1 and E2F2 do not, and this interaction is likewise dependent on the marked box domain (Giangrande et al. 2003). These interactions allow the complexes to bind to target gene promoters that contain E2F sites and either YY1 or E box elements and synergistically activate transcription of these genes.

To further investigate the mechanism underlying the specificity of E2F1 apoptotic function, we have utilized a yeast two-hybrid screen to identify proteins that interact specifically with the E2F1 marked box domain. This screen yielded Jab1 as an E2F-specific binding protein. Jab1 was originally identified as a specificity factor for the c-Jun and JunD transcription factors and has since been shown to interact with many proteins, including p2 $7^{\mathrm{Kip}}$, p53, HIF-1 $\alpha$, Bcl3, LFA-1, MIF, and PR (Claret et al. 1996; Tomoda et al. 1999; Chamovitz and Segal 2001). Jab1 (CSN5) is also a subunit of the COP9 signalosome (CSN), an eight-subunit complex involved in modulating gene transcription, signal transduction, and protein stability (Cope and Deshaies 2003; Wei and Deng 2003). We now demonstrate that Jab1 is essential for E2F1-mediated apoptosis induction.

\section{Results \\ Identification of Jab1 as an E2F1-specific interacting protein}

To explore the mechanism for specificity of E2F1 function in apoptosis, focusing on a potential role for the marked box domain, we identified proteins that interact with the marked box and adjacent region of E2F1 (corresponding to amino acids 252-386) by two-hybrid analysis. We cloned the E2F1 marked box and adjacent region in-frame to the GAL4 DNA-binding domain, separating them with a 14-amino-acid linker. To later compare interaction of the recovered two-hybrid protein between E2F1 and E2F3, we also cloned the marked box and adjacent region of E2F3 into the GAL4 DNA-binding domain vector separated by the same short linker (Fig. 1A). We screened $2.4 \times 10^{6}$ transformants from a human thymus two-hybrid library and recovered 12 different E2F1interacting proteins. These clones were retransformed into a MAT $\alpha$ strain and mated to MATa yeast transformed with GAL4 DBD, GBD-E2F1 MB, GBD-E2F3 MB, or GBD-Cdc6 (full-length). Successfully mated diploids were selected based on their auxotrophic markers (-LU). We next compared the robustness of growth on selective (-AH) media of the mated strains based on two-hybrid interaction. These strength of interaction assays allowed us to categorize interactions into three classes: E2F1specific binding proteins, proteins that bind E2F3 much more strongly than E2F1, and those that bind both the E2F1 and E2F3 marked box regions.

The clones that interacted specifically with E2F1 by two-hybrid analysis included Jab1/CSN5, MCRS1 (Microspherule protein), E1F1 (translation initiation factor), FHOD1 (FH1/FH2 domain-containing protein), Filamin, and SUI1 (Table 1). HSP86 interacted more strongly with the E2F3 marked box and adjacent region than with E2F1. Proteins that bound equivalently strongly to both E2F1 and E2F3 marked box are A-FABP (Adipose fattyacid-binding protein), MX1 (Myxovirus resistance pro-
A

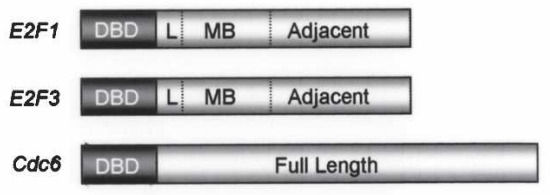

B

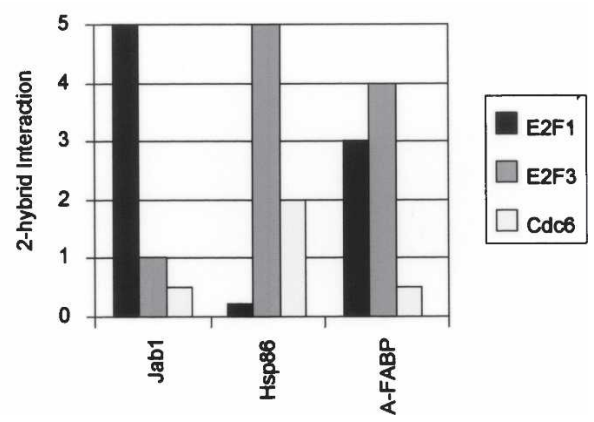

Figure 1. Jab1 interacts with the E2F1 marked box domain. (A) Structure of two-hybrid bait proteins. Graphical representation of bait plasmids used to identify E2F1-specific two-hybrid-interacting proteins. (DBD) Yeast GAL4 DNA-binding domain; (L) 14-amino-acid linker separating the DBD from the E2F bait; (MB) "marked box" regions of E2F1 or E2F3; (Adjacent) region between the marked box and C-terminal transactivation/Rb-binding domain. Amino acids 252-386 of E2F1 and 305-410 of E2F3 were used in these two-hybrid fusion genes. (B) Two-hybrid interaction assays. Jab1, HSP86, and A-FABP are three of the 12 clones recovered from the two-hybrid library as E2F1-interacting proteins. "2-hybrid interaction" refers to the comparative strength of growth on selective media of diploid strains containing either GAL4-E2F1, GAL4-E2F3, or GAL4-Cdc6 and the library-interacting plasmid. 
Table 1. Identification of proteins interacting with the E2F1 marked box domain

\begin{tabular}{llc}
\hline Clone & \multicolumn{1}{c}{ Description } & $\begin{array}{c}\text { E2F } \\
\text { specificity }\end{array}$ \\
\hline Jab1/CSN5 & $\begin{array}{c}\text { c-Jun-activating-binding protein; } \\
\text { COP9 signalosome subunit 5 }\end{array}$ & E2F1 \\
MCRS1 & Microspherule protein & E2F1 \\
E1F1 & Translation initiation factor & E2F1 \\
FHOD1 & FH1/FH2 domain-containing protein & E2F1 \\
Filamin & Actin cytoskeleton organization & E2F1 \\
SUI1 & Translation initiation factor & E2F1 \\
HSP86 & Heat-shock protein 86 & E2F3 \\
A-FABP & Adipose fatty-acid-binding protein & E2F1/E2F3 \\
MX1 & Myxovirus resistance protein & E2F1/E2F3 \\
RNF2 & Ring finger 2 protein & E2F1/E2F3 \\
PRMT3 & Arginine methyltransferase 3 & E2F1/E2F3 \\
RanBPM & Ran-binding protein & E2F1/E2F3 \\
\hline
\end{tabular}

tein), RNF2 (Ring finger 2), PRMT3 (Arginine methyltransferase 3), and RanBPM (Ran-binding protein). An example of the specificity seen in the two-hybrid assay for interactions with E2F1 is shown in Figure 1B.

In light of the focus on E2F1 transcriptional specificity that might be conferred by a unique protein interaction, we focused on the Jab1 protein. Jab1 was originally identified as a c-Jun activation domain-binding protein that functions to stabilize c-Jun on Ap-1-containing promoters and elevates their transcription. To confirm the interaction we detected by two-hybrid analysis between E2F1 and Jab1 using other methods, we performed GST in vitro binding assays. Equal amounts of bacterially expressed GST and GST-E2F1 (full-length) were bound to glutathione-conjugated Sepharose 4B beads (Pharmacia Biotech) and incubated with ${ }^{35} \mathrm{~S}$-labeled full-length Jab1. Jab1 interacts strongly with GST-E2F1 but not with GST alone (Fig. 2A). To further examine this interaction, GST and GST-Jab1 (human, full-length) were incubated with in vitro translated ${ }^{35} \mathrm{~S}-\mathrm{E} 2 \mathrm{~F} 1$ (full-length). In agreement with the GST-E2F1/Jab1 in vitro interaction, labeled E2F1 interacted strongly with GST-Jab1, but not with GST alone (Fig. 2A).

We next performed coimmunoprecipitation experiments to determine whether an endogenous E2F1/Jab1 complex exists in vivo. Nuclear extracts were prepared from REF52 cells, and endogenous E2F1 protein complexes were immunoprecipitated using the KH95 mouse monoclonal antibody, or mouse IgG as a control, and protein $\mathrm{G}$ beads. Immunoprecipitated complexes were washed and analyzed by SDS-PAGE and immunoblotted with Jab1-recognizing antisera. Treatment with mouse IgG control antibody and protein G failed to immunoprecipitate any detectable Jab1/CSN5. Immunoprecipitating E2F1 with the KH95 monoclonal antibody, however, pelleted both E2F1 and Jab1 (Fig. 2B). These findings indicate that endogenous Jab1/CSN5 interacts with E2F1 in vivo.

To investigate the specificity of the E2F1-Jab1 interaction in mammalian cells, HA-tagged E2F1, E2F2, or E2F3 was cotransfected into HEK293 cells with control vector or $5 \times$-Myc-tagged Jab1. Control immunoblots demonstrated that equivalent levels of HA-E2Fs were expressed in the cells. Myc-Jab1 protein complexes were immunoprecipitated from transfected cell lysates using anti-myc antisera (9E10) and protein G beads. Pelleted complexes were analyzed by SDS-PAGE and immunoblotted using HA antisera to detect which of the activator E2Fs interacted with Jab1. We found that immunoprecipitated Jab1 complexes contain HA-E2F1, but failed to bring down significant levels of E2F2 or E2F3 (Fig. 2C) despite the comparable E2F expression levels (Fig. 2D). Even elevated levels of Myc-Jab1 input (Fig. 2E) and immunoprecipitated Myc-Jabl (Fig. 2C, bottom) in HAE2F2- and HA-E2F3-transfected cells failed to bring down significant E2F2 or E2F3. These findings are consistent with the two-hybrid interaction results, indicat-
A

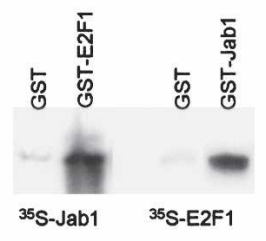

C

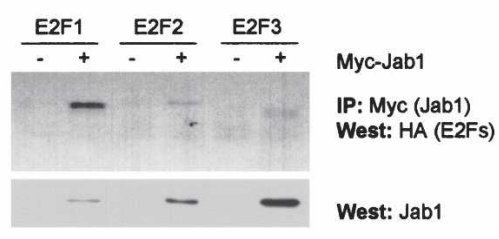

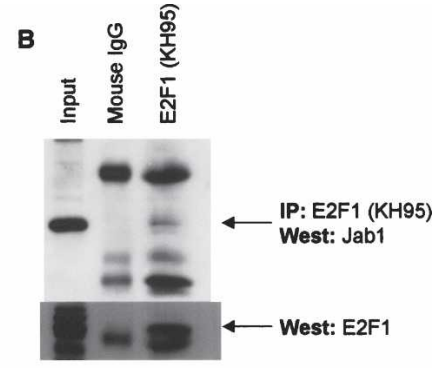

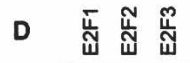

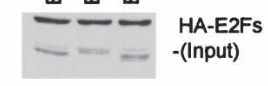

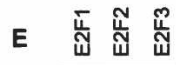

- -Myc-Jab1 (Input)

- Jab1 (endogenous)
Figure 2. Specificity in the interaction of Jab1 with E2F1. (A) Bacterially expressed GST-E2F1 interacts with ${ }^{35}$ S-labeled Jab1 in vitro. Likewise, GST-Jab1 binds with ${ }^{35}$ S-labeled E2F1. (B) Nuclear extracts from REF52 cells were immunoprecipitated with anti-E2F1 antisera (KH95) and protein G beads, separated by SDS-PAGE, and immunoblotted with antiJab1 antisera. In the lower panel, immunoprecipitated E2F1 was detected by immunoblotting with anti-E2F1 antisera (C-20). (C) HEK293 cells were transfected with HA-E2Fs and with pcDNA3 or pcDNA3-5×-Myc-Jab1. Myc-Jab1 complexes were immunoprecipitated with anti-Myc antisera (9E10) and protein $\mathrm{G}$ beads, separated by SDS-PAGE, and immunoblotted with anti-HA antisera. In the lower panel, immunoprecipitated complexes were immunoblotted with anti-Jab1 antisera to assess levels of Jab1 immunoprecipitated. (D) Lysate inputs were immunoblotted for E2F protein levels. $(E)$ Lysates were assayed for Jab1 input levels by immunoblotting. 
ing that Jab1 interacts strongly with the E2F1 but not the E2F3 marked box region.

\section{A role for Jab1 in E2F1-mediated induction of apoptosis and p53 accumulation}

To explore the contribution of Jab1 to E2F1-mediated cell death, we performed apoptosis assays using levels of E2F1 and E2F3 insufficient to induce significant apoptosis on their own. REF52 cells were brought to quiescence through $48 \mathrm{~h}$ of serum deprivation and infected with CMV, HA-E2F1, HA-E2F3 and either with or without Jab1 infection. Jab1 alone caused no apoptosis induction, and expression of E2F1 at low levels led to only a modest induction of apoptosis (Fig. 3A). In contrast, coinfection of Jab1 along with E2F1 resulted in a substantial increase in the number of apoptotic cells (24\%). E2F3, on its own or in combination with Jab1, caused no significant apoptosis. Figure 3B shows that HA-E2F1 and HA-E2F3 were produced at comparable levels in these experiments. These findings together suggest that Jabl combines with E2F1 to induce apoptosis, whereas Jab1 does not appear to contribute to E2F3-induced apoptosis.

Previous work has shown that the E2F1-mediated induction of apoptosis coincides with an induction of p53 accumulation (Wu and Levine 1994; Kowalik et al. 1995; Qin et al. 1995). Given the capacity of Jab1 to cooperate with E2F1 in the induction of apoptosis, we have explored a role for Jab1 in induction of p53 accumulation. Quiescent REF52s were infected with control adenovirus, or adenovirus expressing Jab1, E2F1, or E2F1, and Jab1 at multiplicities of infection (MOIs) equivalent to those used in the apoptosis assays. Cells were harvested $24 \mathrm{~h}$ post-infection for immunoblot analysis with antip53 antisera. As shown in Figure 3C, adenovirus, E2F1, and Jab1 alone fail to induce p53 accumulation. It is important to note that the levels of E2F1 used here (MOI: 20) do not induce p53 on their own, whereas higher levels of E2F1 (MOI: 75) do induce p53 accumulation. These reduced levels of E2F1 significantly induce p53, however, when coinfected with Jab1.

The induction of p53 by E2F1 involves in part an ability of E2F1 to activate the p19 ${ }^{\mathrm{ARF}}$ gene (DeGregori et al. 1997; Bates et al. 1998). ARF then inactivates the E3 ubiquitin ligase $\mathrm{Mdm} 2$, resulting in an accumulation of p53 (Honda and Yasuda 1999). We used RNase protection to detect changes in p19 $\mathrm{ARF}$ and PCNA RNA levels affected by E2F1 and Jab1. REF52 cells were deprived of serum for $48 \mathrm{~h}$, infected with adenovirus expressing E2F1 or Jab1, and RNA was harvested $24 \mathrm{~h}$ post-infection. Lane 1 of Figure 3D contains no added RNA, only probe that has been treated with RNase. We detect low levels of p19ARF levels in quiescent Refs that are unaffected by the addition of Jab1 (Fig. 3D). Adenovirus infection by
A

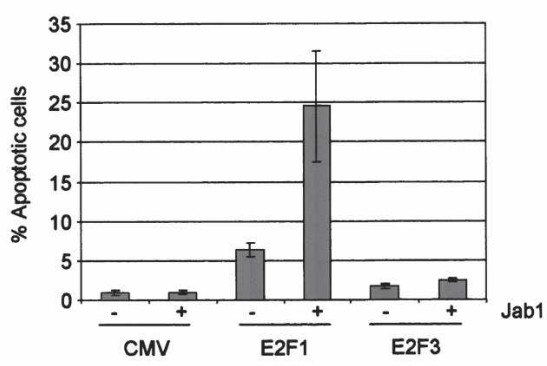

D

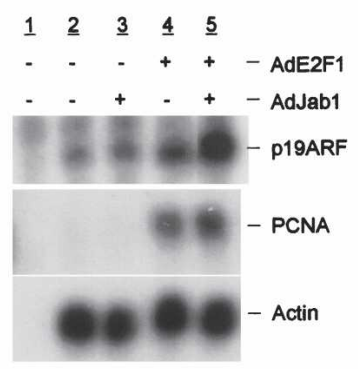

B

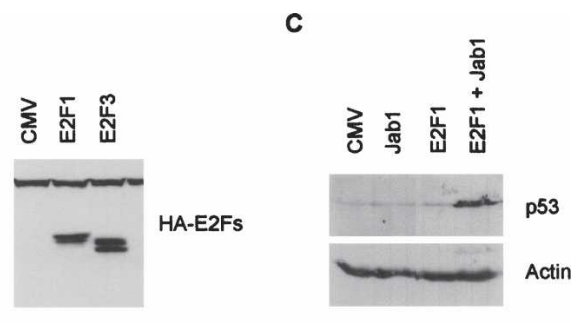

E

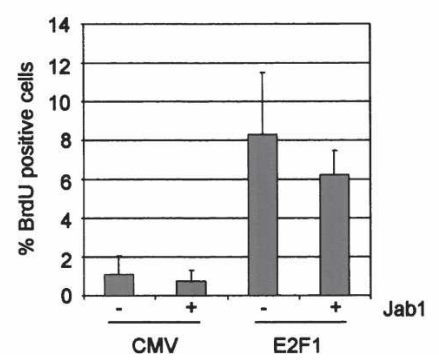

Figure 3. Cooperative induction of apoptosis and p53 accumulation by E2F1 and Jab1. (A) Quiescent REF52 cells were infected with AdCMV, AdE2F1, or AdE2F3 along with either control adenovirus or AdJab1. Cells were harvested at $40 \mathrm{~h}$ post-infection and processed for active caspase-3 levels by FACS analysis. (B) Input levels of E2Fs used in $A$ were determined to be comparable by immunoblotting with anti-HA antisera. $(C)$ Protein extracts from adenovirus-infected REF52 cells were prepared $24 \mathrm{~h}$ post-infection and immunoblotted with p53 and actin-specific antisera. $(D)$ RNase protection assays. REF52 cells were infected with AdE2F1 or AdJab1 as noted, and RNA was isolated at $24 \mathrm{~h}$. RNA was incubated with either a p19 ${ }^{\mathrm{ARF}}$, PCNA, or actin control ${ }^{32} \mathrm{P}$-labeled riboprobe, digested with RNase $\mathrm{A} / \mathrm{T} 1$, and separated on a denaturing polyacrylamide gel. Lane 1 contains no added RNA, only probe and RNase. (E) Quiescent REF52 cells were infected with AdCMV or AdE2F1 along with either control adenovirus or AdJab1. Cells were labeled with BrdU and processed at $24 \mathrm{~h}$ post-infection for BrdU incorporation assays. 
low levels of E2F1 (MOI: 20) failed to cause appreciable p19 ${ }^{\mathrm{ARF}}$ accumulation (Fig. 3D, cf. lanes 4 and 2). However, coinfection with both E2F1 and Jab1 resulted in a significant increase in p19 ${ }^{\mathrm{ARF}}$ expression (Fig. 3D, cf. lanes 5 and 4). We did not detect PCNA in quiescent control infected cells (Fig. 3D, lane 2), and addition of Jab1 did not cause detectable levels of PCNA to accumulate (Fig. 3D, lane 3). E2F1 infection (MOI: 20) caused significant PCNA expression (Fig. 3D, lane 4). Interestingly, coinfection of Jab1 with E2F1 failed to further enhance accumulation of PCNA RNA (Fig. 3D, cf. lanes 5 and 4). This suggests that Jab1 might contribute to the activation of some, but not all, E2F1 target genes. The actin control demonstrates that comparable levels of RNA were used in these assays.

To determine whether Jab1 binding also contributes to E2F1-mediated proliferation, we infected quiescent REF52 cells (at the same MOIs used for the apoptosis experiments) and measured BrdU incorporation. We find that neither CMV nor Jab1 induced significant BrdU incorporation alone $(\sim 1 \%$ score positive). E2F1-infected cells display BrdU incorporation in $\sim 8 \%$ of cells. Coinfection with Jab1 and E2F1 fails to enhance E2F1-depen- dent BrdU incorporation; $\sim 6 \%$ of the doubly infected cells score positive for BrdU incorporation. These findings demonstrate that Jab1 enhances E2F1-dependent apoptosis but not DNA synthesis measured by BrdU incorporation.

The marked box domain of E2F1 is essential for Jab1 interaction and cooperative function

Jab1 was recovered through a two-hybrid interaction assay as binding to the E2F1 marked box and adjacent region located between amino acids 252-386. To further define the Jab1 binding site on E2F1 and to determine whether direct binding between E2F1 and Jab1 is required for apoptosis induction, we performed additional binding/activity studies using a series of chimeric E2F1/ E2F3 proteins. The E2F chimeras used for these studies have been described previously (Hallstrom and Nevins 2003) and are displayed in Figure 4A. The chimera 111331 is composed of the E2F1 cyclin A-binding, DNAbinding, DP1 dimerization, and transactivation/Rb-binding domains, but with the marked box and adjacent region originating from E2F3. The reverse chimera,

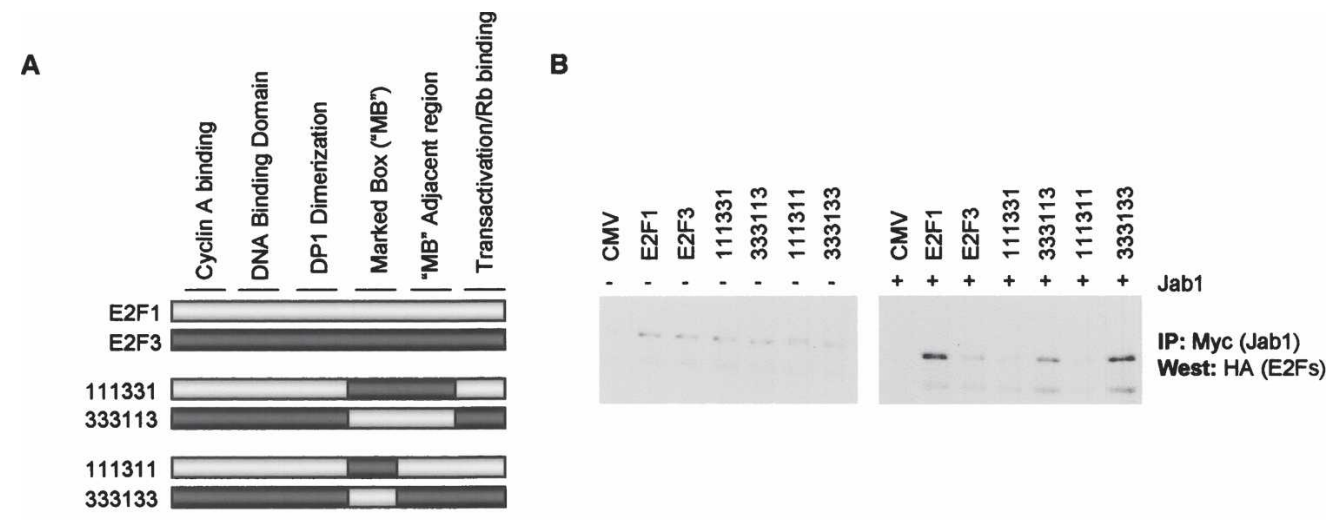

C

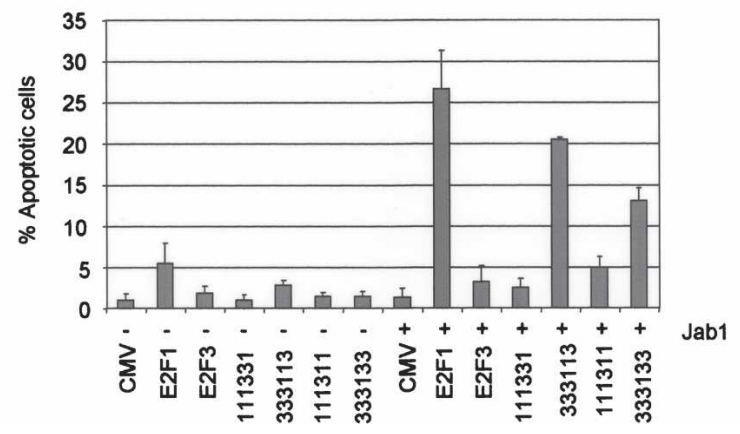

Figure 4. The marked box region of E2F1 is required for Jab1 binding and enhancement of apoptosis. $(A)$ Diagram of E2F chimeras used in these studies. The chimera 111331 is composed of the E2F1 cyclin A-binding, DNA-binding, DP1 dimerization, and transactivation/ Rb-binding domains; however, the marked box and adjacent region originate from E2F3. The reverse chimera, 333113, is E2F3 with the E2F1 marked box and adjacent regions replacing the homologous sequences from E2F3. The chimeras 111311 and 333133 substitute only the marked box region between the genes. $(B)$ HEK293 cells were transfected with HA-E2Fs and with pcDNA3 or pcDNA3-5xMyc-Jab1. Myc-Jab1 complexes were immunoprecipitated with anti-Myc antisera (9E10) and protein G beads, separated by SDS-PAGE, and immunoblotted with anti-HA antisera. $(C)$ Quiescent REF52 cells were infected with AdCMV, AdE2F1, AdE2F3, or adenovirus expressing chimeric E2Fs along with either control adenovirus or AdJab1. Cells were harvested at 40 h post-infection and processed for active caspase-3 levels by FACS analysis. 
333113, is E2F3 with the E2F1 marked box and adjacent regions replacing the homologous sequences from E2F3. The chimeras 111311 and 333133 have swapped only the marked box region between these two genes. Each of these chimeras, along with E2F1 and E2F3 as controls, were tested for their ability to bind to Jab1 by coimmunoprecipitation assays and whether the interaction coincided with enhanced apoptosis induction. Full-length E2F1 interacts with Jab1 by immunoprecipitation (Fig. 4B) and synergizes with Jab1 to induce apoptosis (Fig. 4C), whereas E2F3 displays neither of these properties. The chimera 333113 contains the E2F1 marked box and adjacent region sequences that were used in the twohybrid screen to detect an interaction between E2F1 and Jab1. We find that this chimera binds to Jabl and induces significant apoptosis (comparable to full-length E2F1) in the presence of Jab1. Substitution of the E2F1 marked box and adjacent regions with the homologous sequences from E2F3 blocks its binding to Jab1 and likewise impairs its responsiveness by Jab1 to induce apoptosis. To further define the binding site of Jab1 on E2F1, we assessed binding by Jab1 to the E2F chimeras with only the marked box region substituted (chimeras 111311 and 333133). We find that substituting the E2F1 marked box sequences with the homologous region from E2F3 abolishes Jab1 binding and apoptosis induction. The reverse chimera, 333133, which contains only E2F1 marked box sequences, binds to Jab1, and, furthermore, Jabl enhances the apoptotic potential of this chimera. These findings strongly indicate that Jabl binds to the marked box region of E2F1 and that this binding contributes to apoptosis induction. Mutated E2F1 chimeras that substitute marked box sequences with sequences originating from E2F3 abolish both Jab1 binding and also Jab1 enhancement of E2F1-dependent apoptosis induction.

\section{Jab1 is required for E2F1-mediated apoptosis and $p 53$ induction}

If Jab1 functions as a specificity factor for E2F1-induced apoptosis, depleting cells of Jab1 by short hairpin RNA (shRNA) should impair the ability of E2F1 to induce apoptosis. We targeted Jab1 for shRNA-mediated degradation based on previously reported Jab1 knockdown studies (Groisman et al. 2003). We cloned two rat-specific knockdown sequences into the pSuper.retro vector and generated shJab1 retrovirus to stably infect REF52 cells. In total, we generated three independent shJab1 knockdown cell lines in REF52 cells using the shJab1 pSuper.retro construct \#1. Two of these lines were generated by transfecting shJab1 pSuper.retro into BD Amphotropic packaging retroviral cell lines and then infecting REF52s (shJab1-1, shJab1-3). The other was generated using $\varnothing$ ecotropic packaging cell lines (shJab1-2). We found that shJab1 construct \#1 effectively degraded Jab1 levels (Fig. $5 \mathrm{~A}, \mathrm{~B}$, lanes 2,31 , but a second targeting construct failed to reduce Jab1 protein levels (shJab1-4; Fig. 5B, lane 4). Jab1 and the CSN complex in which Jab1/CSN5 resides influence the proteasomal degradation of some proteins. One possibility is that reducing Jab1/CSN5 levels might
Figure 5. E2F1-mediated induction of apoptosis and p53 accumulation requires Jab1. (A) ShJabl targeting sequences were cloned in the pSuper.retro plasmid and transfected into BD amphotropic packaging cells. Packaged retrovirus was used to stably infect REF52 cells (the resultant line is referred to here as shJab1-1). E2F1 and actin protein levels are unaffected by depletion of Jab1 levels in REF52 cells. (B) Additional shJab1 REF52 cell lines were generated by stably infecting with shJabl constructs packaged in Ø-ecotropic lines (shJab1-2) and an amphotropic line (shJab1-3). ShJab1-4 are REF52 cells infected with a different shJab1 targeting construct that failed to degrade Jab1. Extracts from these cells were immunobloted for Jab1 levels. (C) Wild-type REF52 cells, REFs targeted for shJab1 degradation (shJab1-1, shJab1-2, shJab1-3), and REFs stably infected with the nonfunctional shJab1 construct (shJab1-4) were brought to quiescence and infected with E2F1 adenovirus (MOI: 75). Floating and adherant cells were harvested at $40 \mathrm{~h}$ post-infection and analyzed for apoptosis levels by measuring the percentage of cells with active, cleaved caspase-3. The results shown here are the percentage of apoptosis compared with the percentage of apoptotic cells seen in wild-type cells. The level of apoptosis in wild-type cells was set to $100 \%$ to display the percent drop in apoptosis levels in mutant cells compared with levels in wild-type cells. $(D)$ Wild-type and shJab1 REF52 cells were brought to quiescence and infected with control or Myc-expressing adenovirus. Floating and adherant cells were harvested $40 \mathrm{~h}$ post-infection to measure activated caspase- 3 levels by flow cytometry. (E) Wild-type and shJab1-1 REF52 cells were infected with control or E2F1-expressing adenovirus (MOI:75) and harvested $24 \mathrm{~h}$ post-infection for immunoblotting with p53, PCNA, and actin-specific antisera.
A

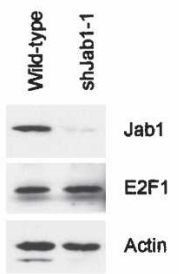

D

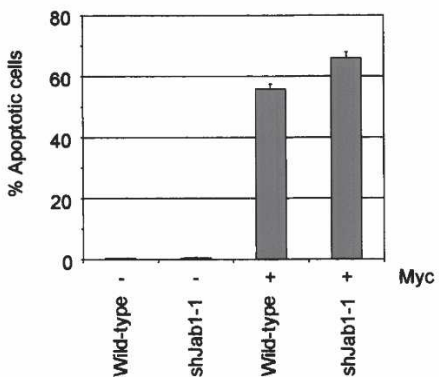

c
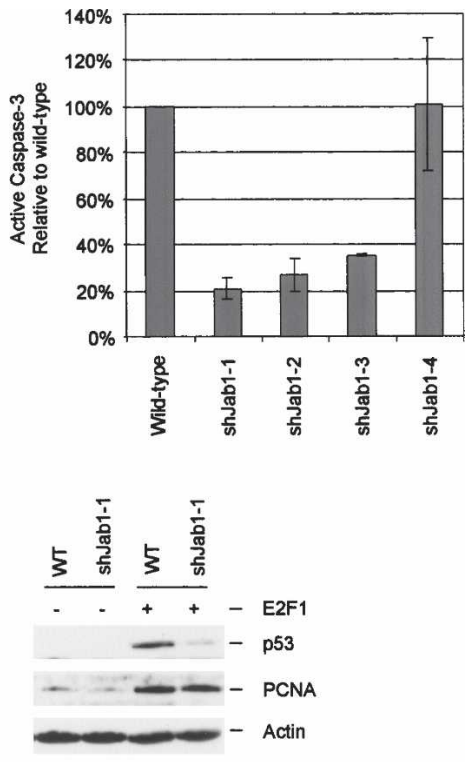
influence E2F1 activity indirectly by changing its steadystate levels. We find that reducing Jabl levels, however, does not influence endogenous E2F1 levels (Fig. 5A).

We performed caspase- 3 cleavage apoptosis assays to determine whether the loss of Jab1 impaired E2F1-induced apoptosis. Wild-type REF52, the three REF52 cell lines depleted of Jab1 (shJab1-1, shJab1-2, shJab1-3), and the cell line infected with the nonfunctional shJabl vector (shJab1-4) were brought to quiescence before infecting with Ad-E2F1 and measuring apoptosis levels. Results of the experiments are shown in Figure 5C and are reported as a ratio of percent caspase-3-positive shJab1 cells relative to the percent of caspase-3-positive wildtype REF52 cells. Wild-type apoptosis has been set to $100 \%$ to facilitate comparison among the different lines. Each of the four cell lines depleted of Jab1 shows a large reduction in E2F1-induced apoptosis compared with wild-type REFs, with reductions ranging from $65 \%$ to $80 \%$. Importantly, shJab1-4 cells carrying the shJab1 construct that does not degrade Jab1 showed no defect in E2F1-induced apoptosis. These findings indicate that E2F1-induced apoptosis is impaired in cells depleted of Jab1.

To determine the extent to which Jab1 might contribute to a distinct apoptosis-inducing pathway, we explored whether Myc-induced apoptosis is impaired in cells depleted of Jab1. Myc, like E2F1, is an oncogene that induces apoptosis via similar caspase-3-dependent mitochondrial pathways and induces some of the same apoptotic target genes as E2F1. However, as shown in Figure 5D, shRNA-mediated depletion of Jab1 has no effect on Myc-induced apoptosis ( $60 \%$ in both wild-type and shJab1 cells). This finding suggests that the contribution of Jab1 to apoptosis is specific to its binding to E2F1 and is not through activation of downstream apoptotic pathways.

We also explored whether the ability of E2F1 to induce p53 accumulation was dependent on Jab1. Wild-type and shJab1-1 cells were infected with CMV or E2F1-expressing adenovirus, and p53/PCNA protein levels were compared by immunoblotting. CMV induces no detectable p53 expression in wild-type or shJab1-1 cells (Fig. 5E). AdE2F1 (MOI: 75) induces strong p53 accumulation in wild-type cells. This E2F1 induction of p53 is significantly impaired, however, in cells lacking Jab1. The E2F target gene PCNA is induced by E2F1 in wild-type quiescent REF52s. Interestingly, the loss of Jab1 does not compromise E2F1's capacity to induce PCNA. This further strengthens the notion that the E2F1/Jabl complex may regulate some E2F targets (p19 $\mathrm{ARF} / \mathrm{p} 53)$ but not others (PCNA).

Restoration of E2F1-mediated apoptosis by Jab1, independent of deneddylating function

To verify that impaired E2F1-induced apoptosis was due specifically to the loss of Jab1, we restored Jab1 levels in these cells using a form of the gene that is not degraded by the shRNA construct. We introduced mutations into the Jab1 cDNA that do not affect the Jab1 protein sequence but do prevent its recognition and degradation by shRNA-targeting sequences. We also introduced two point mutations (H138A/H140A) into the nondegradable Jab1 cDNA construct to eliminate the deneddylation enzymatic motif in Jab1/CSN5. These nondegradable versions of Jab1 were cloned into the retroviral vector pMSCVhyg and stably infected into shJab1-1 REF52 cells. We compared Jab1 expression levels in cell extracts from wild-type, shJab1-1 cells, and shJab1-1 cells complemented with the nondegradable forms of Jab1, either with or without the mutated deneddylation enzymatic motif. We found that both nondegradable forms of Jab1 partially restored Jab1 protein levels (Fig. 6A). We tested these four lines for the ability of E2F1 to induce apopto-
A

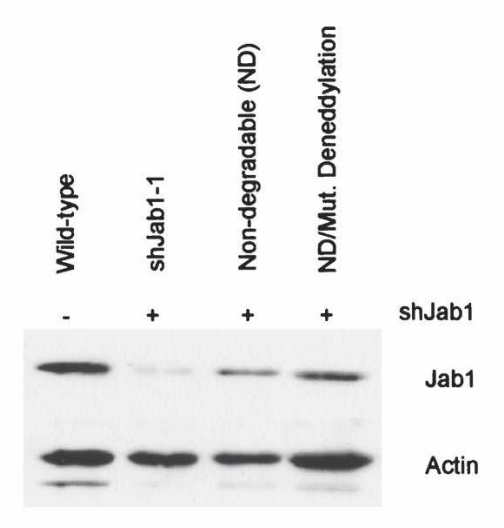

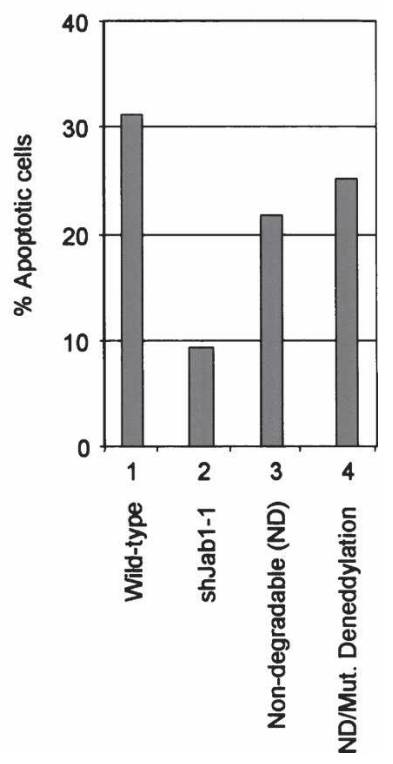

Figure 6. Restoration of E2F1-mediated apoptosis by Jab1, independent of deneddylating function. (A) REF52 cells already targeted for Jab1 shRNA degradation (shJab1-1) were stably infected with a nondegradable version of Jab1, or the nondegradable form with (H138A, H140A) amino acid substitution mutations that eliminates Jab1/CSN5 Nedd8 isopeptidase activity (mutant deneddylation). Extracts were analyzed by immunoblotting with anti-Jabl and anti-actin antisera. $(B)$ Cells described in $A$ were brought to quiescence, infected with AdE2F1, and harvested for apoptosis assays at $40 \mathrm{~h}$ post-infection. 
sis. We find that replenishing Jab1 levels restores E2F1induced apoptosis (Fig. 6B, lane 3). Complementing Jab1deficient cells with the deneddylation-defective allele of Jab1 likewise nearly fully restored E2F1-induced apoptosis (Fig. 6B, lane 4). This suggests that the deneddylating activity of Jab1/CSN5 is not essential for Jab1's contribution to E2F1-induced apoptosis.

\section{Discussion}

The Rb/E2F pathway plays a central role in the decisions that link cellular proliferation with cell fate determination. A large number of studies now show that the apoptotic pathway involving p53 is directly linked to the $\mathrm{Rb} / \mathrm{E} 2 \mathrm{~F}$ pathway, likely providing a mechanism to eliminate cells that have sustained mutations in $\mathrm{Rb}$ or other genes that control E2F accumulation or activity (Sherr 1998). Various studies point to E2F1 as the stronger effector of the apoptotic signal associated with deregulation of the Rb/E2F pathway. Furthermore, E2F1, but not E2F2 or E2F3, is targeted by DNA damage-induced signals. These culminate in ATM/ATR or Chk2 phosphorylation of E2F1, leading to accumulation through enhanced protein stability (Lin et al. 2001; Stevens et al. 2003). Considering that E2F1 can serve as both a positive activator of cellular proliferation as well as an inducer of cell death, this E2F family member can be viewed as a key determinant in the decisions of cellular fate. As such, the basis by which E2F1 specificity is achieved becomes critical to understanding the process of balancing the proliferative response with the cell death response. The work presented here now points to a role for Jab1 as a determinant of this specificity.

Jab1 has been linked to a complex and diverse list of interacting proteins and cellular functions. Jab1 was originally described as a c-Jun/JunD coactivator (Claret et al. 1996). Jab1 can regulate cell cycle proliferation by promoting the nuclear export and proteasomal degradation of the cell cycle inhibitor p27Kip1 (Tomoda et al. 1999). Jab1 is also known as CSN5, the fifth subunit (out of eight total) of the CSN complex. This complex is conserved from yeast to humans and plays a critical role in cellular signaling pathways and organism development (Chamovitz and Segal 2001). CSN-complexed CSN5 is a Nedd8 isopeptidase that binds Skp1-Cullin/CDC53-FBox (SCF) and cleaves (deneddylates) Nedd8-Cul1 conjugates, inactivating SCF and its regulated proteasomal degradation of particular targets/Cope and Deshaies 2003). Gene disruption of Jab1/Csn5 is embryonic lethal, and day $6.5 \mathrm{Jab1}^{-/-}$embryos exhibit elevated cyclin E, p27, and p53 protein levels and increased apoptosis (Tomoda et al. 2004). It is unclear whether these effects of Jab1 loss of function are E2F-dependent or whether they result from disruption of the full CSN, which can promote p53 degradation through direction phosphorylation (Bech-Otschir et al. 2001). Mice lacking signalosome components Csn2 (Lykke-Andersen et al. 2003), Csn3 (Yan et al. 2003), or the SCF pathway genes Uba3 (Tateishi et al. 2001) or Cull (Dealy et al. 1999; Wang et al. 1999) display similar activation of cyclin E and p53 levels and embryonic lethality at the same developmental stage, indicating that disabled CSN-Nedd8-SCF signaling may be responsible for these effects. It is important to note that the E2F1-dependent apoptosis rescued by Jab1/Csn5 does not require the deneddylation enzymatic motif present in Jab1 to elicit this response. Our results would indicate that Jab1/Csn5 can regulate p53 protein levels by different mechanisms, either indirectly through E2F1-dependent activation or through degradation instigated by the full CSN. It is interesting to note that CSN5/Jab1 can reside in both the CSN-complexed and monomeric forms in mammalian cells (Kwok et al. 1998; Oron et al. 2002; Gusmaroli et al. 2004). Taken together, this variety of studies suggests a complex array of physical interactions and activities for Jab1/CSN5, with different functions inside and outside the CSN, and it is possible that the equilibrium between these two forms is regulated by physiologic signaling events.

Our previous work has demonstrated that the capacity of E2F1 to specifically induce apoptosis, relative to the other E2F family members, is dependent on the marked box domain. Other work has implicated the E2F marked box domain in mediating protein interactions that dictate specificity of promoter recognition through an ability to interact with other promoter-specific transcription factors. For example, E2F2 and E2F3a have been shown to interact with the RYBP protein that provides a bridge between E2Fs and the YY1 transcription factor, facilitating binding of this complex to the Cdc6 promoter (Schlisio et al. 2002). In addition, E2F3 has also been shown to interact with the E-box transcription factor TFE3, and again this is dependent on the marked box domain (Giangrande et al. 2003). Significantly, because these interactions are restricted to particular E2F family members, they provide a basis for specificity of transcription control within the E2F family. Previous work has identified unique protein interactions involving E2F1 that influence its transcriptional activity. For example, TopBP1 binds the N-terminal region of E2F1, but not other E2Fs, and represses E2F1-induced apoptosis (Liu et al. 2003). Likewise, the Ets-family transcription factor GABP- $\gamma$ binds exclusively to E2F1 and suppresses E2F1-induced apoptosis (Hauck et al. 2002). To date, however, no E2F1specific binding factor has been shown to enhance E2F1induced apoptosis. The work we present here now implicates the Jab1 protein as a specific partner for E2F1, providing specificity of gene activation in the context of induction of apoptosis.

Since there is no evidence for a role for Jabl in directly binding DNA, we do not know at the present time if the function as an E2F1 cofactor is to facilitate promoter recognition or to perform some alternate function that provides specificity for gene regulation by E2F1. Certainly, the observation that the interaction of Jab1 with E2F1 involves the marked box domain of E2F1, which has been shown to mediate interactions of other E2F proteins that provide for promoter specificity, suggests this as a possible mechanism for a role for Jab1. But, without knowledge of what other promoter-specific transcription factor might be involved, or what the critical promoter elements might be, we have no direct evi- 
dence for such a mechanism. Nevertheless, the data presented here do implicate Jab1 as a specificity factor that allows E2F1 to stimulate an apoptotic response distinct from a role in mediating cell proliferation.

\section{Materials and methods}

\section{Plasmid construction}

GBDU-E2F1 and GBDU-E2F3 plasmids used in the two-hybrid analysis were generated as follows: Sequences from E2F1 (amino acids 252-386) and E2F3 (amino acids 305-410), followed by an introduced "TGA" stop codon, were PCR amplified and cloned in-frame into the GAL4 DNA-binding domain expression plasmid pGBDU-C1. E2F sequences were separated from the GAL4 DBD by a cDNA encoding a protein linker GAACTAAAAAC TGCTGCTTTGGCTCAACATGCGCTCGAGAAG that encodes the protein linker ELKTAALAQHALEK. GBDU-Cdc6 (full-length) was a gift of Jean Cook (University of North Carlina, Chapel Hill, NC). GST-E2F1 is cloned as full-length human E2F1 in pGEX-2TK. Full-length human Jab1 was PCR amplified from an IMAGE clone and cloned as a BamHI/NotI fragment into pcDNA3. GST-Jab1 was generated by PCR amplifying full-length human Jabl and cloning in-frame as a BamHI/NotI fragment into the vector pGEX-6P1. 5x-Myc-Jab1 was generated by PCR amplifying full-length human Jabl and cloning in-frame as a BamHI/NotI fragment into pcDNA3 already containing $5 \times-$ Myc sequence. Ha-E2F1 (pcDNA3), HaE2F2 (pcDNA3), and Ha-E2F3 (pRcCMV) are full-length human cDNAs and have been described previously (Lin et al. 2001). Plasmids for p19 $\mathrm{ARF}, \mathrm{PCNA}$, and actin riboprobe generation were constructed by isolating RNA from REF52 cells and using RT-PCR to clone fragment into Bluescript KSII+. The specific regions of each gene amplified and cloned are nucleotides 27227 (p19ARF), 232-433 (PCNA), and 79-291 (actin). Sequences targeted by Jab1 shRNA constructs \#1 and \#2 were based on studies that successfully knocked down Jabl in human cells (Groisman et al. 2003). The oligo Rat sh\#1 top primer gatcccc GCGCAGAGTATCGATGAAAttcaagagaTTTCATCGATACT CTGCGCtttttggaaa was annealed to Rat sh\#1 bottom primer agcttttccaaaaaGCGCAGAGTATCGATGAAAtctcttgaaTTTCAT CGATACTCTGCGCggg, and the Rat sh\#2 top primer gatc cccCATGCAGGAAGCGCAGAGTttcaagagaACTCTGCGCT TCCTGCATGtttttggaaa was annealed to Rat sh\#2 bottom primer agcttttccaaaaaCATGCAGGAAGCGCAGAGTtctcttgaa ACTCTGCGCTTCCTGCATGggg and cloned into pSuper. retro plasmid as BglII/HindIII fragments. The capitalized letters in the oligos refer to the specific 19 nucleotides in Rat Jab1 targeted for shRNA-mediated degradation. The shRNA-resistant form of Jabl was constructed by introducing point mutations into the human Jab1 cDNA in the region targeted for degradation. Specifically, the 19 nucleotides targeted by shRNA were mutated from gcgcagagtatcgatgaaa (wild-type) to gcCca ATCGatTgaCgaGa (resistant) using the GeneEditor mutagenesis system (Promega). Mutations are shown in capital letters and do not result in altered protein sequence. This shRNAresistant form of Jab1 was further mutagenized to inactivate its deneddylation enzymatic motif. Specifically, the codons at amino acid positions 138 and 140 were mutated from cat to GCt (138) and cac to GCc (140), respectively. Both of these mutations replaced histidines with alanines at both positions. Both of these shRNA-resistant clones, with and without the deneddylation mutation, were cloned into the retroviral packaging vector pMSCVhyg (Clontech). Each of the plasmids generated for these studies was sequenced to ensure that no mutations occurred during PCR amplification.
Cell culture, apoptosis assays, and measurement of DNA synthesis

REF52 cells were brought to quiescence by plating $\left(3 \times 10^{5}\right.$ cells $/$ $60-\mathrm{mm}$ plate) in serum containing media for $24 \mathrm{~h}$ and then washing and replacing with $0.25 \%$ serum-containing media. Cells were deprived of serum for $48 \mathrm{~h}$ before infecting with adenovirus. Cell numbers were determined prior to infection to infect with equal adenoviral multiplicities. Floating and adherent cells were harvested at $40 \mathrm{~h}$ post-infection, fixed with formaldehyde, stained with a PE-conjugated antibody that binds to active, cleaved caspase- 3 cleavage, and analyzed by FACS following the manufacturer's protocol (BD PharMingen). For cell proliferation assays, cells were labeled immediately post-infection with $50 \mu \mathrm{M}$ BrdU for $24 \mathrm{~h}$. Cells were formaldehyde fixed, DNA denatured with $1.5 \mathrm{~N} \mathrm{HCl}$, incubated with anti-BrdU antibody (Amersham Pharmacia-RPN 202), PBS washed, treated with Texas Red conjugated anti-mouse secondary (1:2000) for 1 $\mathrm{h}$, washed, and DAPI stained. A total of at least 300 nuclei taken from multiple fields chosen at random were counted for each sample.

\section{Adenovirus and retrovirus cloning}

Adenovirus expressing HA-tagged E2F1 and E2F3 and chimeric E2Fs was constructed and described previously (Hallstrom and Nevins 2003). Human Jab1-expressing adenovirus was generated by cloning the human Jabl cDNA into the pShuttle vector and following the adEasy adenovirus construction protocol. Adenoviral titers were determined by indirect immunofluorescence against the viral Mr 72,000 E2 gene product. Titers for all adenovirus used in these studies were determined simultaneously to avoid experimental variation. Retroviruses were packaged using Ø-ecotropic (Nolan lab) or amphotropic packaging cells from BD Biosciences (BD AmphoPack-293). Plasmid DNA (4 $\mu \mathrm{g})$ was transfected into the packaging line (in $1200 \mu \mathrm{L}$ DMEM) using Fugene 6 transfection reagent (Roche). After $24 \mathrm{~h}$, another $1000 \mu \mathrm{L}$ of media was added to the cells, and they were incubated another $20 \mathrm{~h}$. Supernatant was filtered through 0.45 $\mu \mathrm{m}$ HT Tuffryn membrane low-protein-binding filter syringes ( $44 \mathrm{~h}$ post-transfection) and used to infect target cells. Polybrene $(8 \mu \mathrm{g} / \mathrm{mL})$ was included to assist infection efficiency. Drug selection was started $24 \mathrm{~h}$ post-infection. Drug selection concentrations for REF52 cells were $1.25 \mu \mathrm{g} / \mathrm{mL}$ puromycin, $300 \mu \mathrm{g} / \mathrm{mL}$ G418, or $50 \mu \mathrm{g} / \mathrm{mL}$ hygromycin.

\section{Two-hybrid analysis}

A two-hybrid library derived from human thymus cDNAs (pACT2) was obtained from Clontech and amplified. Two-hybrid analysis was performed according to protocols outlined by James et al. (1996). The yeast strain pJ69-4A was transformed with the GBDU-E2F1 marked box bait construct and selected. This transformed strain was transformed at high efficiency with the human thymus two-hybrid library. We recovered $\sim 2.4 \times 10^{6}$ colonies, which was seven times the representation of the library. Transformants were plated on selective media lacking adenine or media lacking histidine but containing $3 \mathrm{mM} 3$-aminotriazole to select transformants with library plasmids that bind E2F1. Transformants were cured of URA3 bait plasmid on 5-floro-orotic acid (FOA)-containing plates. Library plasmids from these strains were isolated and retransformed into a MAT $\alpha$ strain. These retransformed strains were mated with a MATa strain-transformed vector, GBD-E2F1 bait, GBD-E2F3 bait, or full-length Cdc6 bait. Successfully mated strains containing bait and putative library-interacting plasmid were tested again on selective media to verify interaction phenotype and also to compare interaction strength to E2F3 and Cdc6 relative to E2F1. 


\section{Protein interaction and immunoblot assays}

For endogenous Jab1/E2F1 immunoprecipitations, nuclear extracts were prepared from REF52 cells by harvesting cells into PBS, resuspending in lysis buffer (10 mM HEPES at $\mathrm{pH} 7.9,10$ $\mathrm{mM} \mathrm{KCl}, 0.1 \mathrm{mM}$ EDTA, $0.1 \mathrm{mM}$ EGTA) for $20 \mathrm{~min}$ on ice. Cells were futher lysed by adding $25 \mu \mathrm{L}$ NP-40 (10\%), vortexing for $10 \mathrm{sec}$, and centrifuging. Pellet (nuclei) was resuspended and lysed in IP buffer (50 mM HEPES at pH 7.3, $150 \mathrm{mM} \mathrm{NaCl}, 2.5$ mM EGTA, 10\% glycerol, 0.1\% Tween-20, $1 \mathrm{mM} \mathrm{NaF}, 1 \mathrm{mM}$ DTT, $0.1 \mathrm{mM}$ Na3VO3, and Sigma Complete protease inhibitors. Supernatant was precleared with protein G beads (Roche) for $30 \mathrm{~min}$, centrifuged, and coupled to $5 \mu \mathrm{g}$ anti-E2F1 antisera (KH95) for 60 min. Twenty microliters of protein G beads (Roche) was added to supernatant and rotated for $60 \mathrm{~min}$. Beads were washed three times with IP buffer. Protein complexes were resuspended in $2 \times$ SDS sample buffer, separated on a protein gel, and analyzed by immunoblotting. For coimmunoprecipitations of Jab1 and E2F chimeras, HEK293 cells were transfected with individual HA-tagged E2Fs alone or with 5x Myc-tagged Jab1. Transfected cells were harvested after $24 \mathrm{~h}$ into lysis buffer $(10$ mM HEPES at pH 7.9, $400 \mathrm{mM} \mathrm{NaCl}, 0.1 \mathrm{mM}$ EDTA, 5\% glycerol, $1 \mathrm{mM}$ DTT, $20 \mu \mathrm{M}$ N-ethylmaleimide, $1 \mathrm{mM}$ PMSF, and Sigma Complete protease inhibitors). Cells were spun, resuspended in lysis buffer, rotated for $30 \mathrm{~min}$ at $4^{\circ} \mathrm{C}$, and centrifuged twice to remove supernatant from cell debris. Supernatant was precleared with protein $\mathrm{G}$ beads (Roche) for $60 \mathrm{~min}$, centrifuged, and coupled to $3 \mu \mathrm{g}$ anti-Myc antisera (9E10) for $60 \mathrm{~min}$. Twenty microliters of protein $\mathrm{G}$ beads (Roche) was added to supernatant and rotated for $60 \mathrm{~min}$. Beads were washed three times with wash buffer $(20 \mathrm{mM}$ Tris- $\mathrm{HCl}$ at $\mathrm{pH} 7.4,1 \mathrm{mM}$ EDTA, 10\% glycerol, $150 \mathrm{mM} \mathrm{NaCl}, 0.1 \%$ Triton X-100, $1 \mathrm{mM} \mathrm{DTT}$, and Sigma Complete protease inhibitors). Protein complexes were resuspended in $2 \times$ SDS sample buffer, separated on a protein gel, and analyzed by immunoblotting. GST interaction assays were performed as described earlier (Giangrande et al. 2003). Immunoblots were performed as previously described (Hallstrom and Nevins 2003). Antibodies used were Jab1 (Zymed, 34-3000), E2F1 (Santa Cruz C-20, SC-193; KH95, SC-251), Myc epitope (Santa Cruz 9E10, SC-40), HA (Santa Cruz Y-11, SC-805), p53 (Santa Cruz FL-393, SC-6243), actin (Santa Cruz C-2, SC-8432), and PCNA (Santa Cruz PC10, SC-56).

\section{RNase protection assays}

Riboprobes were generated by reverse transcribing linearized plasmids $(3 \mu \mathrm{g})$ with ${ }^{32} \mathrm{P}$-rUTP. RNA was isolated from infected REF52 cells 24 h post-infection using an RNeasy kit (Qiagen) and homogenized using QIAshredder. RNase protection assays were performed using the RPAIII kit following manufacturer's instructions (Ambion).

\section{Acknowledgments}

We thank Kaye Culler for assistance in the preparation of the manuscript. T.C.H. was supported by an American Cancer Society postdoctoral fellowship (PF-02-004-01-CCG).

\section{References}

Attwooll, C., Denchi, E.L., and Helin, K. 2004. The E2F family: Specific functions and overlapping interests. $E M B O ~ I . ~ 23:$ 4709-4716.

Bates, S., Phillips, A.C., Clark, P.A., Stott, F., Peters, G., Ludwig, R.L., and Vousden, K.H. 1998. p14ARF links the tumour suppressors RB and p53. Nature 395: 124-125.

Bech-Otschir, D., Kraft, R., Huang, X., Henklein, P., Kapelari, B., Pollmann, C., and Dubiel, W. 2001. COP9 signalosome-specific phosphorylation targets p53 to degradation by the ubiquitin system. EMBO J. 20: 1630-1639.

Chamovitz, D.A. and Segal, D. 2001. JAB1/CSN5 and the COP9 signalosome. A complex situation. EMBO Rep. 2: 96-101.

Claret, F.X., Hibi, M., Dhut, S., Toda, T., and Karin, M. 1996. A new group of conserved coactivators that increase the specificity of Ap-1 transcription factors. Nature 383: 453-457.

Cope, G.A. and Deshaies, R.J. 2003. COP9 signalosome: A multifunctional regulator of SCF and other cullin-based ubiquitin ligases. Cell 114: 663-671.

Croxton, R., Ma, Y., Song, L., Haura, E.B., and Cress, W.D. 2002. Direct repression of the Mcl-1 promoter by E2F1. Oncogene 21: 1359-1369.

Dealy, M.J., Nguyen, K.V., Lo, J., Gstaiger, M., Krek, W., Elson, D., Arbeit, J.M., Kipreos, E.T., and Johnson, R.S. 1999. Loss of Cull results in early embryonic lethality and dysregulation of cyclin E. Nat. Genet. 23: 245-248.

DeGregori, J. 2002. The genetics of the E2F family of transcription factors: Shared functions and unique roles. Biochim. Biophys. Acta 1602: 131-150.

DeGregori, J., Leone, G., Miron, A., Jakoi, L., and Nevins, J.R. 1997. Distinct roles for E2F proteins in cell growth control and apoptosis. Proc. Natl. Acad. Sci. 94: 7245-7250.

Denchi, E.L. and Helin, K. 2005. E2F1 is crucial for E2F-dependent apoptosis. EMBO Rep. 6: 661-668.

Dyson, N. 1998. The regulation of E2F by pRB-family proteins. Genes \& Dev. 12: 2245-2262.

Field, S.J., Tsai, F.-Y., Kuo, F., Zubiaga, A.M., Kaelin Jr., W.G., Livingston, D.M., Orkin, S.H., and Greenberg, M.E. 1996. E2F-1 functions in mice to promote apoptosis and suppress proliferation. Cell 85: 549-561.

Giangrande, P.H., Hallstrom, T.C., Tunyaplin, C., Calame, K., and Nevins, J.R. 2003. Identification of the $\mathrm{E}$ box factor TFE3 as a functional partner for the E2F3 transcription factor. Mol. Cell. Biol. 23: 3707-3720.

Groisman, R., Polanowska, J., Kuraoka, I., Sawada, J., Saijo, M., Drapkin, R., Kisselev, A.F., Tanaka, K., and Nakatani, Y. 2003. The ubiquitin ligase activity in the DDB2 and CSA complexes is differentially regulated by the COP9 signalosome in response to DNA damage. Cell 113: 357-367.

Gusmaroli, G., Feng, S., and Deng, X.W. 2004. The Arabidopsis CSN5A and CSN5B subunits are present in distinct COP9 signalosome complexes, and mutations in their JAMM domains exhibit differential dominant negative effects on development. Plant Cell 16: 2984-3001.

Hallstrom, T.C. and Nevins, J.R. 2003. Specificity in the activation and control of transcription factor E2F-dependent apoptosis. Proc. Natl. Acad. Sci. 100: 10848-10853.

Hauck, L., Kaba, R.G., Lipp, M., Dietz, R., and von Harsdorf, R. 2002. Regulation of E2F1-dependent gene transcription and apoptosis by the ETS-related transcriptional factor GABPg1. Mol. Cell. Biol. 22: 2147-2158.

Honda, H. and Yasuda, H. 1999. Association of p19(ARF) with Mdm2 inhibits ubiquitin ligase activity of Mdm2 for tumor suppressor p53. EMBO J. 18: 22-27.

Humbert, P.O., Verona, R., Trimarchi, J.M., Rogers, C., Dandapani, S., and Lees, J.A. 2000. E2f3 is critical for normal cellular proliferation. Genes \& Dev. 14: 690-703.

Irwin, M., Martin, M.C., Phillips, A.C., Seelan, R.S., Smith, D.I., Liu, W., Flores, E.R., Tsai, K.Y., Jacks, T., Vousden, K.H., et al. 2000. Role for the p53 homologue p73 in E2F-1-induced apoptosis. Nature 407: 645-648.

Jacks, T., Fazeli, A., Schmitt, E.M., Bronson, R.T., Goodell, 
M.A., and Weinberg, R.A. 1992. Effects of an Rb mutation in the mouse. Nature 359: 295-300.

James, P., Halladay, J., and Craig, E.A. 1996. Genomic libraries and a host strain designed for highly efficient two-hybrid selection in yeast. Genetics 144: 1425-1436.

Kowalik, T.F., DeGregori, J., Schwarz, J.K., and Nevins, J.R. 1995. E2F1 overexpression in quiescent fibroblasts leads to induction of cellular DNA synthesis and apoptosis. J. Virol. 69: 2491-2500.

Kowalik, T.F., DeGregori, J., Leone, G., and Nevins, J.R. 1998. E2F1-specific induction of apoptosis and p53 accumulation is modulated by mdm2. Cell Growth Differ. 9: 113-118.

Kwok, S.F., Solano, R., Tsuge, T., Chamovitz, D.A., Ecker, J.R., Matsui, M., and Deng, X.W. 1998. Arabidopsis homologs of a c-Jun coactivator are present both in monomeric form and in the COP9 complex, and their abundance is differentially affected by the pleiotropic cop/det/fus mutations. Plant Cell 10: 1779-1790.

Lee, E.Y.-H.P., Chang, C.-Y., Hu, N., Wang, Y.-C.J., Lai, C.-C., Herrup, K., Lee, W.-H., and Bradley, A. 1992. Mice deficient for $\mathrm{Rb}$ are nonviable and show defects in neurogenesis and haematopoiesis. Nature 359: 288-294.

Leone, G., DeGregori, J., Yan, Z., Jakoi, L., Ishida, S., Williams, R.S., and Nevins, J.R. 1998. E2F3 activity is regulated during the cell cycle and is required for the induction of $S$ phase. Genes \& Dev. 12: 2120-2130.

Lin, W.-C., Lin, F.-T., and Nevins, J.R. 2001. Selective induction of E2F1 in response to DNA damage, mediated by ATMdependent phosphorylation. Genes \& Dev. 15: 1833-1845.

Lissy, N.A., Davis, P.K., Irwin, M., Kaelin, W.G., and Dowdy, S.F. 2000. A common E2F-1 and p73 pathway mediates cell death induced by TCR activation. Nature 407: 642-644.

Liu, K., Lin, F.T., Ruppert, J.M., and Lin, W.-C. 2003. Regulation of E2F1 by BRCT domain-conting protein TopBP1. Mol. Cell. Biol. 23: 3287-3304.

Lykke-Andersen, K., Schaefer, L., Menon, S., Deng, X.W., Miller, J.B., and Wei, N. 2003. Disruption of the COP9 signalosome Csn2 subunit in mice causes deficient cell proliferation, accumulation of p53 and cyclin E, and early embryonic death. Mol. Cell. Biol. 23: 6790-6797.

Moroni, M.C., Hickman, E.S., Denchi, E.L., Caprara, G., Colli, E., Cecconi, F., Muller, H., and Helin, K. 2001. Apaf-1 is a transcriptional target for E2F and p53. Nat. Cell Biol. 3: 552558.

Nahle, Z., Polakoff, J., Davuluri, R.V., McCurrach, M.E., Jacobson, M.D., Narita, M., Zhang, M.Q., Lazebnik, Y., Bar-Sagi, D., and Lowe, S.W. 2002. Direct coupling of the cell cycle and cell death machinery by E2F. Nat. Cell Biol. 4: 859-864.

Nevins, J.R. 1998. Toward an understanding of the functional complexity of the E2F and Retinoblastoma families. Cell Growth Differ. 9: 585-593.

Oron, E., Mannervik, M., Rencus, S., Harari-Steinberg, O., Neuman-Silberberg, S., Segal, D., and Chamovitz, D.A. 2002. COP9 signalosome subunits 4 and 5 regulate multiple pleiotropic pathways in Drosophila melanogaster. Development 129: 4399-4409.

Pan, H., Yin, C., Dyson, N.J., Harlow, E., Yamasaki, L., and van Dyke, T. 1998. Key roles for E2F1 in signaling p53-dependent apoptosis and in cell division within developing tumors. Mol. Cell 2: 283-292.

Qin, X.-Q., Livingston, D.M., Kaelin, W.G., and Adams, P.D. 1994. Deregulated transcription factor E2F-1 expression leads to S-phase entry and p53-mediated apoptosis. Proc. Natl. Acad. Sci. 91: 10918-10922.

Qin, X.-Q., Livingston, D.M., Ewen, M., Sellers, W.R., Arany, Z., and Kaelin Jr., W.G. 1995. The transcription factor E2F-1 is a downstream target of RB action. Mol. Cell. Biol. 15: 742-755.

Rogoff, H.A., Pickering, M.T., Debatis, M.E., Jones, S., and Kowalik, T.F. 2002. E2F1 induces phosphorylation of p53 that is coincident with p53 accumulation and apoptosis. Mol. Cell. Biol. 22: 5308-5318.

Rogoff, H.A., Pickering, M.T., Frame, F.M., Debatis, M.E., Sanchez, Y., Jones, S., and Kowalik, T.F. 2004. Apoptosis associated with deregulated E2F activity is dependent on E2F1 and Atm/Nbs1/Chk2. Mol. Cell. Biol. 24: 2968-2977.

Saavedra, H.I., Wu, L., de Bruin, A., Timmers, C., Rosol, T.J., Weinstein, M., Robinson, M.L., and Leone, G. 2002. Specificity of E2F1, E2F2, and E2F3 in mediating phenotypes induced by loss of Rb. Cell Growth Differ. 13: 215-225.

Schlisio, S., Halperin, T., Vidal, M., and Nevins, J.R. 2002. Interaction of YY1 with E2Fs, mediated by RYBP, provides a mechanism for specificity of E2F function. EMBO J. 21: 5775-5786.

Shan, B. and Lee, W.-H. 1994. Deregulated expression of E2F-1 induces S-phase entry and leads to apoptosis. Mol. Cell. Biol. 14: 8166-8173.

Sherr, C.J. 1998. Tumor surveillance via the ARF-p53 pathway. Genes \& Dev. 12: 2984-2991.

Stevens, C., Smith, L.G., and La Thangue, N.B. 2003. Chk2 activates E2F-1 in response to DNA damage. Nat. Cell Biol. 5: 401-409.

Stiewe, T. and Putzer, B.M. 2000. Role of the p53 homologue p73 in E2F1-induced apoptosis. Nat. Genet. 26: 464-469.

Tateishi, K., Omata, M., Tanaka, K., and Chiba, T. 2001. The NEDD8 system is essential for cell cycle progression and morphogenetic pathway in mice. J. Cell Biol. 155: 571-579.

Tomoda, K., Kubota, Y., and Kato, J. 1999. Degradation of the cyclin-dependent-kinase inhibitor p27Kip1 is instigated by Jab1. Nature 398: 160-165.

Tomoda, K., Yoneda-Kato, N., Fukumoto, A., Yamanaka, S., and Kato, J.-Y. 2004. Multiple functions of Jab1 are required for early embryonic development and growth potential in mice. J. Biol. Chem. 279: 43013-43018.

Tsai, K.Y., Hu, Y., Macleod, K.F., Crowley, D., Yamasaki, L., and Jacks, T. 1998. Mutation of E2f-1 suppresses apoptosis and inappropriate $\mathrm{S}$ phase entry and extends survival of $\mathrm{Rb}$ deficient mouse embryos. Mol. Cell 2: 293-304.

Wang, Y., Penfold, S., Tang, X., Hattori, N., Riley, P., Harper, J.W., Cross, J.C., and Tyers, M. 1999. Deletion of the Cull gene in mice causes arrest in early embryogenesis and accumulation of cyclin E. Curr. Biol. 9: 1191-1194.

Wei, N. and Deng, X.W. 2003. The COP9 signalosome. Annu. Rev. Cell Dev. Biol. 19: 261-286.

Wu, X. and Levine, A.J. 1994. p53 and E2F-1 cooperate to mediate apoptosis. Proc. Natl. Acad. Sci. 91: 3602-3606.

Wu, L., Timmers, C., Maiti, B., Saavedra, H.I., Sang, L., Chong, G.T., Nuckolls, F., Giangrande, P., Wright, F.A., Field, S.J., et al. 2001. The E2F1-3 transcription factors are essential for cellular proliferation. Nature 414: 457-462.

Yamasaki, L., Bronson, R., Williams, B.O., Dyson, N.J., Harlow, E., and Jacks, T. 1998. Loss of E2F-1 reduces tumorigenesis and extends the lifespan of $\mathrm{Rb1}^{\mathrm{r} /-}$ mice. Nat. Genet. 18: 360-364.

Yan, J., Walz, K., Nakamura, H., Carattini-Rivera, S., Zhao, Q., Vogel, H., Wei, N., Justice, M.J., Bradley, A., and Lupski, J.R. 2003. COP9 signalosome subunit 3 is essential for maintenance of cell proliferation in the mouse embryonic epiblast. Mol. Cell. Biol. 23: 6798-6808.

Ziebold, U., Reza, T., Caron, A., and Lees, J.A. 2001. E2F3 contributes both to the inappropriate proliferation and to the apoptosis arising in Rb mutant embryos. Genes \& Dev. 15: 386-391. 


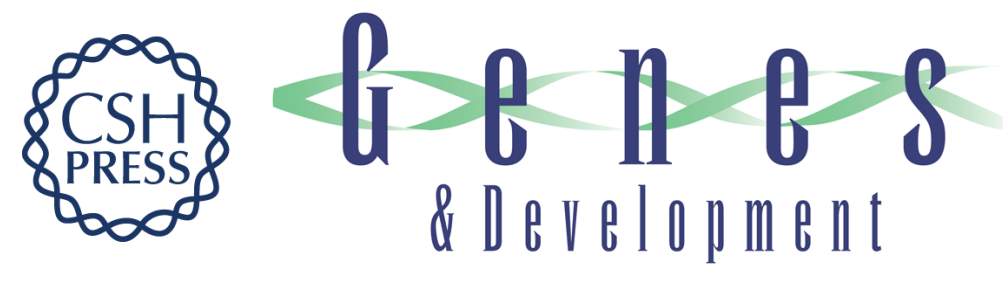

\section{Jab1 is a specificity factor for E2F1-induced apoptosis}

Timothy C. Hallstrom and Joseph R. Nevins

Genes Dev. 2006, 20:

Access the most recent version at doi:10.1101/gad.1345006

References This article cites 58 articles, 32 of which can be accessed free at: http://genesdev.cshlp.org/content/20/5/613.full.html\#ref-list-1

License

Email Alerting Receive free email alerts when new articles cite this article - sign up in the box at the top Service right corner of the article or click here.

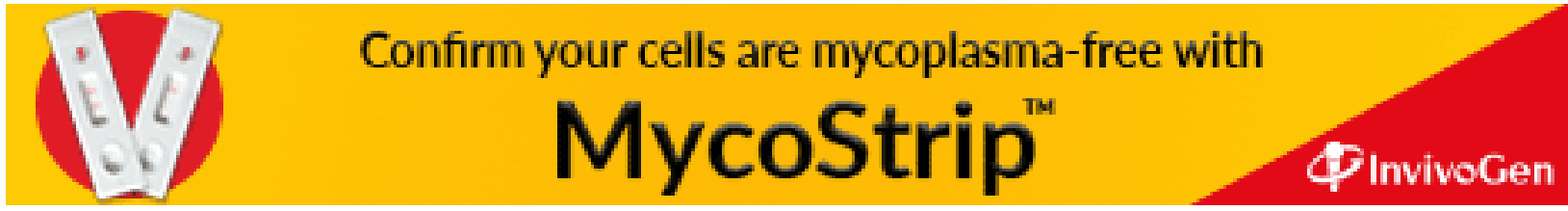

